\title{
INSTRUCTIONS FOR AUTHORS FOR PREPARATION OF CAMERA-READY MANUSCRIPTS
}

\author{
Ari Viljanen, Risto Pirjola and Heikki Nevanlinna \\ Finnish Meteorological Institute \\ Geophysical Research Division \\ P.O.B. 503, FIN-001010 Helsinki, Finland \\ ari.viljanen@fmi.fi
}

\begin{abstract}
The purpose of these instructions is to help you prepare your camera-ready summary for the abstract book. These instructions are printed using the desired format.
\end{abstract}

\section{GUIDELINES}

All abstracts scheduled for presentation at the meeting will be published in the Finnish Meteorological Institute Reports series. The publication will be printed by offset directly from the typescript. Abstracts will appear exactly as they are submitted.

Your contribution should be in English and consist of two A4 pages in length, including all figures and tables. The recommended font is Times (12 points). Leave one empty line between the address and the abstract. The abstract title should be centered in bold (all caps). Below title center name(s) of the author(s) with affiliation, complete mailing address and the email address of the first author. Left and right justify is recommended. Set all margins to $30 \mathrm{~mm}$. Use single line space and separate the paragraphs by an empty line. For references, please use the practice of Viljanen et al. (1999). Only black and white figures are allowed. Do not insert page numbers.

Flush headings left in all caps. Position figures and tables at the page top or bottom; avoid placing them in the middle. Figure captions should be below the figures and table captions above the tables.

Save your abstract as a PostScript or PDF file and send it as an email attachment to Ari Viljanen (ari.viljanen@fmi.fi), or give an FTP or WWW address from where the file can be fetched. In all cases, print your abstract on paper and check the hardcopy carefully! Indicate in your email which session you prefer, and whether you like to give an oral or poster presentation.

\section{REFERENCES}

Viljanen, A., O. Amm and R. Pirjola, 1999: Modelling Geomagnetically Induced Currents During Different Ionospheric Situations. Journal of Geophysical Research, 104, 28059-28072. 